\title{
State-of-the-art Electron Microscopy in Medical Mineralogy and Biogeochemistry
}

\author{
Ruggero Vigliaturo ${ }^{1}$, Reto Gieré ${ }^{1,2}$
}

${ }^{1}$ Department of Earth and Environmental Science, University of Pennsylvania, 240 S. 33rd Street, Hayden Hall, Philadelphia, PA 19104-6316, U.S.A

ruggero.vigliaturo@gmail.com; giere@sas.upenn.edu

${ }^{2}$ Center of Excellence in Environmental Toxicology,

University of Pennsylvania, Philadelphia, PA

The advent of new atomic-resolution microscopes allows for correction and stabilization of the $5^{\text {th }}$ order aberrations. This aberration correction leads to a resolution of $\leq 1.6 \AA$ at voltages as low as $30 \mathrm{kV}$.

The combination of extremely low voltages, atomic resolution, and double-EDX and Dual-EELS detectors push the analytical limits towards unprecedented scales.

In this brief review, we will show how different techniques may be used to understand the evolution of nanominerals interacting with simulated biofluids, cell cultures and/or bacteria.

We will demonstrate an extensive application of DualEELS at different voltages and electron doses with the aim to define the coordination geometry and valence state of metals that may generate reactive oxygen species in biological materials (e.g., $\mathrm{Fe}$ and $\mathrm{Cr}$ ). The study of the low-loss spectrum was used to determine the chemical state of lighter elements, such as $\mathrm{C}$, and Si porosity.

Finally, we will lay the foundation for innovative in-situ dissolution experiments with toxic nanoparticles in simulated biofluids. Such in-situ studies inside an atomic-resolution microscope will be performed to track nanoparticle mass loss and valence-state variations in real-time.

The collected information will not be useful only for understanding the fundamental interaction but also for determining the boundary conditions within which biochemical reactions take place Determination of these boundary conditions will help the medical community to improve the understanding of toxic pathway mechanisms. 